\title{
Modelling and Application of Signal Decoupling of Adjacent Channels of a Whole-Roller Seamless Flatness Meter
}

\author{
Huaxin YU, ${ }^{1)}$ Dongcheng WANG, ${ }^{1,2) *}$ Hongmin LIU, ${ }^{1,2)}$ Tongyuan ZHANG $^{1)}$ and Lipo YANG ${ }^{1,2)}$ \\ 1) National Engineering Research Center for Equipment and Technology of Cold Strip Rolling, Yanshan University, \\ Qinhuangdao, 066004 China. \\ 2) State Key Laboratory of Metastable Materials Science and Technology, Yanshan University, Qinhuangdao, 066004 China.
}

(Received on August 2, 2019; accepted on November 15, 2019; J-STAGE Advance published date: January 18, 2020)

\begin{abstract}
For ultra-thin and super-wide cold rolled strips, the flatness problem are still very conspicuous. To ensure that the surface of a strip is not scratched, a whole-roller seamless flatness meter (abbreviated as WRS flatness meter) is developed. Due to the structure of the measuring roll, which has cylindrical holes under its surface, both the signal of the measuring channels and the signal of the adjacent channels will be significantly interfered when a load is applied to a WRS flatness meter, causing flatness measurement errors. To eliminate mutual interference between channels, flatness measuring principle and channel coupling mechanism are analyzed, and the concept and model of coupling coefficient are proposed. Then, some examples are given to illustrate significant errors caused by coupling, which demonstrates the necessity of decoupling the channels. Coupling coefficients between the channels are obtained by experimental calibration, and interference between the channels is eliminated with a decoupling matrix equation. Through simulation and industrial applications, it is shown that the theoretical model proposed in this paper realizes decoupling of the channels of the WRS flatness meter, which improves flatness detection and controlling accuracy.
\end{abstract}

KEY WORDS: cold rolled strip; flatness meter; whole-roller seamless measuring roll; channel coupling coefficient; decoupling matrix equation.

\section{Introduction}

Cold rolled strips are widely used in food packaging, automotive, home appliances, electrical devices, electronics, rail transit, aerospace and other industries. Flatness is an important quality factor for cold rolled strips. Online flatness measurement and control is the core technology of high-precision strip cold rolling mills and is the premise of realizing closed-loop control of the flatness. Due to technical difficulties, flatness meters have long been monopolized by a few companies, such as ABB Stressometer from Sweden, ${ }^{1)}$ SIFLAT developed by SIEMENS and flatness measurement systems developed by BFI in Germany, ${ }^{2)}$ Optical hot rolling shape measuring device developed by Belgium Metallurgical Research Center, Laser flatness meter with translation image method developed by French Iron and Steel Research Institute. ${ }^{3)}$ A large number of international scholars have also been carrying out theoretical and experimental studies on flatness measure and control. A novel contactless stress detection technology based on a magnetoresistance sensor and the magnetoelastic effect, enabling the detection of internal stress in manufactured cold-rolled strips, was presented by Ben Guan et al. ${ }^{4}$ High-resolution full-field

\footnotetext{
* Corresponding author: E-mail: wangdongcheng@ysu.edu.cn DOI: https://doi.org/10.2355/isijinternational.ISIJINT-2019-472
}

measurements were used to measure the wrinkling shape and thermal field by Dinh Cuong Tran et al. ${ }^{5)}$ A new flatness meter which employed LED dot pattern projection method was developed by Yoshito ISEI et al. ${ }^{6}$ ) The application of a steady state elastic-viscoplastic finite element model was described by S. Abdelkhalek et al. ${ }^{7)}$ Based on the theoretical analysis and the practical engineering, a new high-precision flatness detection model and a new strip shape control model were established to analyse the problem of strip edge shape and achieve the synchronizing control of complex shape defects by Lipo Yang et al. ${ }^{8,9)}$ In the past 10 years, Yanshan University has made a breakthrough in the research of cold rolled strip flatness meters. A compact wireless embedded signal processor for cold rolling strip flatness meter and a new type of cold strip entire roller inlayed block intelligence flatness meter for on-line detection were developed. ${ }^{10,11)}$ Yanshan University and Anshan Iron and Steel Co., Ltd. cooperatively developed a new generation of whole roll wireless flatness meter and intelligent flatness control system. ${ }^{12)}$ Flatness control models and methods were summarized, which provided a new direction for the breakthrough of improving the shape quality using the new type flatness meters and flatness control systems. ${ }^{13}$ ) The latest developed flatness meter has also been successfully applied in industrial applications.

In recent years, with the unceasing development of cold 
rolled strips and their downstream industries, users have increasingly strict requirements on surface quality of strips, especially for fine strips. As gaps between adjacent outer rings may scratch strips, segmented flatness rolls are not suitable for rolling finished strips and nonferrous metal strips during smoothing and finishing processes. To solve this problem, a WRS flatness meter is developed. Since the surface of the measuring roll is a complete and seamless cylindrical surface, it can completely avoid crushing and scratching surface of strips, which is the future development trend of flatness meters.

During flatness measuring process, a flatness meter will be disturbed by vibration, lateral temperature differences in the strip, measuring roll installation error and deflection deformation. To ensure accuracy of flatness measurement, flatness signal must be denoised and compensated. Scholars have conducted a great deal of research on this topic.

Flatness signal was filtered by a discrete tracking differentiator to eliminate noise interference. ${ }^{14)}$ The wavelet denoising technology was used to preprocess flatness signal, which improved recognition accuracy of flatness. ${ }^{15)}$ To evaluate residual stress profile of strips, a semi-analytical inverse Cauchy problem based on plane complex elasticity with conformal mapping techniques was presented by Daniel Weisz-Patrault for a measuring roll with cylindrical holes. ${ }^{16)}$ Liu Jiawei et al. analyzed the stress of a measuring roll and obtained a deflection model of a measuring roll by using the influence function method. ${ }^{17)} \mathrm{YU}$ Bingqiang et al. analyzed the influences of measurement error of strip edges, transverse temperature difference in strips, deflection of measuring roll and the shape of the strip coil on the flatness measurement results and established a corresponding compensation models. ${ }^{18)} \mathrm{Li}$ Rongmin et al. analyzed the influence of deflection and installation accuracy (precision) of a measuring roll on flatness signal, established a corresponding compensation model, and formulated installation accuracy requirements of a measuring roll. ${ }^{19,20)}$ A position error compensation model of a measuring roll without a guide roll was established by Zhao Zhangxian et al., ${ }^{21)}$ which improved accuracy of flatness control. Yang Lipo et $a .^{22,23)}$ performed an in-depth analysis of influences of zero residual voltage, partial coverage of a channel by strips at the edge of a measuring roll, installation precision of a measuring roll and deflection deformation on flatness signal, and they established a compensation model, which significantly improved accuracy of detection signal and laid a foundation for improving control precision. Temperature field and thermal deformation models of a measuring roll based on heat transfer and thermal elasticity theory were established by WU Haimiao et al. ${ }^{24)}$ Wang Pengfei et al. established an accurate compensation model for partial coverage of strips at the edge a channel of a measuring roll and proposed a method for determining flatness of strips when there is a failure in some channels of a measuring roll. ${ }^{25}$ ) That is, they interpolated flatness signal to ensure accuracy and stability of a control system consequently.

Due to the special structure of the WRS flatness meter, when a load is applied to one of the measuring channels, both the signal of the stressed channel and the signal of the adjacent channels will be significantly interfered, which means that there is a coupling phenomenon between the channels. This coupling phenomenon causes significant errors in flatness measuring and must be decoupled to improve detection accuracy. However, relevant research on this issue has not yet been seen internationally. For this reason, based on the analysis of flatness measuring principle and channels coupling mechanism, a coupling matrix equation and a decoupling matrix equation of the WRS flatness measuring roll are proposed. The entries of the coupling matrix are obtained through experimental calibration. The substantial influence of the coupling phenomenon and the significant effects of the decoupling method are illustrated by examples.

\section{Main Structures of the Flatness Measuring Roll}

According to the structure of a measuring roll, a flatness meter can be divided into several types, such as a segmented type and a whole-roll seamless type. ${ }^{26,27)}$ The Swedish ASEA segmented flatness meter was widely used worldwide. Figure 1 shows that this segmented flatness measuring roll consists of a core shaft, outer rings, and piezomagnetic sensors. Segmented flatness measuring rolls are easier to manufacture than other types. A series of outer rings and sensors are arranged along the axial direction of the measuring roll to detect the pressure $F_{i}$. Then, the values of $F_{i}$ are converted into tension $T_{i}$ by a signal processing computer to obtain a distribution of flatness along the width direction of strips. Because the segmented measuring roll has a gap that is dozens of micrometers wide between every two adjacent roll rings, the measuring channels are basically independent of each other, so there is no interference between adjacent channels. However, gaps between the channels may cause indentations or chromatic aberrations on strips. At present, this kind of measuring roll is suitable only for occasions where surface quality of strips is not high (or for semifinished strips). Segmented measuring rolls are not sufficient to meet surface quality requirements of high-end strip products.

To solve the problems above, a new type of WRS flatness measuring roll ${ }^{10,28)}$ was proposed, as shown in Fig. 2. The measuring roll surface is a complete cylinder with 2-4 axially cylindrical through-holes punched circumferentially under the surface. A series of piezoelectric sensors are mounted side-by-side at same distance in each hole. The distance

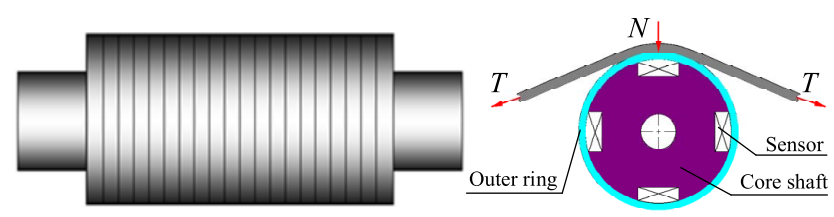

Fig. 1. Segment piezomagnetic type flatness measuring roll. (Online version in color.)

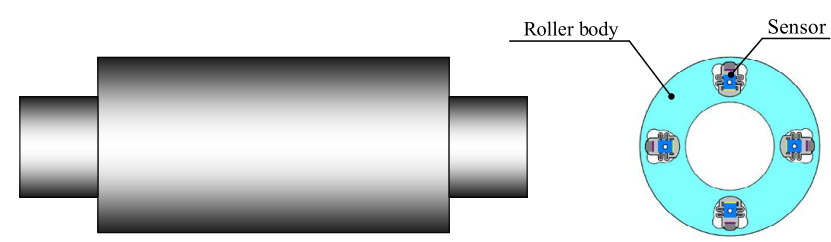

Fig. 2. Whole-roller seamless (WRS) flatness measuring roll. (Online version in color.) 
between the sensors is $26 \mathrm{~mm}$ or $52 \mathrm{~mm}$. The number of holes is determined by the number of signals to be detected when the measuring roll rotates once. The flatness measuring roll is suitable for measuring finished strips because the outer surface is seamless and does not crush or scratch the surface of strips. However, a new problem is generated by this type of roller. The outer surface of the measuring roll is a whole cylinder; Hence, sensors in several channels will be affected by deformation of a certain force, indicating that adjacent channels will be significantly interfered.

\section{Analysis and Modelling of Channels Coupling and Decoupling}

\subsection{Principle of Flatness Measurement}

Figure 3 shows that during flatness measuring process, strip is set above the measuring roll at a certain wrap angle under action of tension, which generates a radial pressure on the measuring roll. When the strip has flatness flaw, the nonuniform distribution of radial pressure along the axial direction can be detected by the shape meter, and the distribution of radial pressure can be converted to the flatness distribution.

Assume the strip thickness is $h$, the width of the strip is $B$, the measuring roll has a total of $\mathrm{n}$ channels, radial pressure of the strip on the roll surface of channel $i$ is $F_{i}$, average value of radial pressure on the roll surface of each channel is $\bar{F}$, the total tension of the strip is $T$. $E$ and $v$ are elastic modulus and Poisson's ratio of the strip, respectively.

I-Unit is used internationally to represent basic unit of flatness of strips. An I-Unit indicates that relative length difference is $10^{-5}$. Then flatness $\varepsilon$ can be expressed as

$$
\varepsilon=-\frac{\left(1-v^{2}\right)}{E} \frac{F_{i}-\bar{F}}{\bar{F}} \frac{T}{B h} \times 10^{5} \quad \text { (I-Unit). }
$$

\subsection{Mechanism Model of Channels Coupling}

The internal structure of the measuring roll is shown in Fig. 4. The brown blocks are piezoelectric sensors, the total number of sensors is $n$, and the thickness of the outer part of the measuring roll is $t$. When the surface of the measuring roll is subjected to a pressure, elastic flattening deformation occurs. The pressure and deformation are transmitted to sensors to generate a linear change in the electric signal, thereby sensing the magnitude of the pressure. The outer part of the WRS flatness roll is a continuous whole. According to the elastic half-space theory, ${ }^{29-31)}$ when a certain channel (chan-

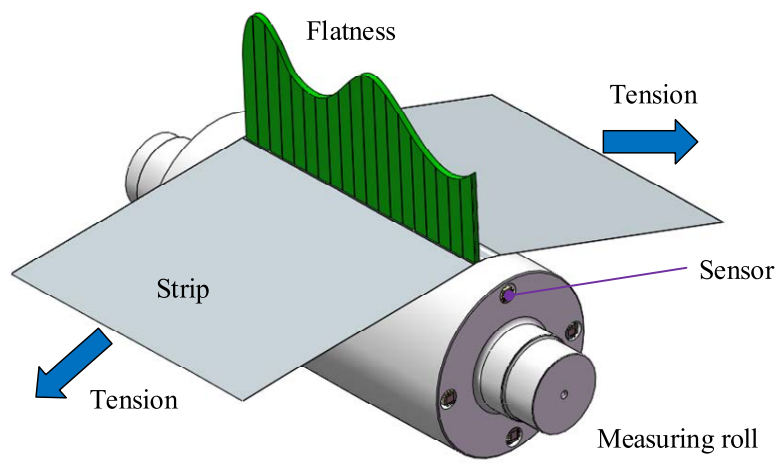

Fig. 3. Schematic diagram of flatness measurement. (Online version in color.) nel j) of the roller surface is subjected to pressure $F_{j}$, it will cause elastic flattening displacement of the outer part of not only the certain channel (purple) but also other channels (green). Hence, even if only channel $j$ is stressed, the other sensors will detect changes in the electrical signal, which is obviously contrary to the expectation that each measuring channel detects only the load applied to its own channel.

When pressure $F_{j}$ is applied to channel $j$, the displacement $u_{i j}$ was generated at the center point (blue point) of the upper surface of channel $i$. This displacement can be expressed as

$$
u_{i j}=\beta_{i j} F_{j} \text {. }
$$

where $\beta_{i j}$ is the influence coefficient. The detailed expression for the influence coefficient ${ }^{30)}$ is

$$
\beta_{i j}=\frac{1-v_{\mathrm{r}}^{2}}{\pi E_{\mathrm{r}} b_{j}}\left\{\begin{array}{l}
\ln \frac{\sqrt{t^{2}+\left[\left(y_{i}-y_{j}\right)+b_{j} / 2\right]^{2}}+\left(y_{i}-y_{j}\right)+b_{j} / 2}{\sqrt{t^{2}+\left[\left(y_{i}-y_{j}\right)-b_{j} / 2\right]^{2}}+\left(y_{i}-y_{j}\right)-b_{j} / 2} \\
+\frac{1}{2\left(1-v_{w}\right)} \ln \frac{\left(y_{i}-y_{j}\right)+b_{j} / 2}{\sqrt{t^{2}+\left[\left(y_{i}-y_{j}\right)+b_{j} / 2\right]^{2}}} \\
-\frac{1}{2\left(1-v_{w}\right)} \ln \frac{\left(y_{i}-y_{j}\right)-b_{j} / 2}{\sqrt{t^{2}+\left[\left(y_{i}-y_{j}\right)-b_{j} / 2\right]^{2}}}
\end{array}\right\} .
$$

where $E_{\mathrm{r}}$ and $v_{\mathrm{r}}$ are elastic modulus and Poisson's ratio of the roll body, respectively.

Assuming that the stiffness of the sensor in channel $i$ is $k_{i}$, the coupling force of channel $i$ produced by the force of channel $j$ is

$$
N_{i j}=k_{i} u_{i j}=k_{i} \beta_{i j} F_{j}=\alpha_{i j} F_{j} .
$$

where $\alpha_{i j}=k_{i} \beta_{i j}=N_{i j} / F_{j}$, which is referred to as the coupling coefficient. In particular, $\alpha_{j j}=1$.

When the surface of the measuring roller is fully loaded along its axial direction, the coupling phenomenon exists in all channels, and the final measured force of channel $i$ is

$$
N_{i}=\sum_{j=1}^{n} N_{i j}=k_{i} \sum_{j=1}^{n} \beta_{i j} F_{j}=\sum_{j=1}^{n} \alpha_{i j} F_{j} .
$$

Equation (5) can be expressed in matrix form as

$$
\left[\begin{array}{c}
N_{1} \\
N_{2} \\
\vdots \\
N_{n-1} \\
N_{n}
\end{array}\right]=\left[\begin{array}{ccccc}
\alpha_{11} & \alpha_{12} & \cdots & \alpha_{1(n-1)} & \alpha_{1 n} \\
\alpha_{21} & \alpha_{21} & \cdots & \alpha_{2(n-1)} & \alpha_{2 n} \\
\vdots & \vdots & \vdots & \vdots & \vdots \\
\alpha_{(n-1) 1} & \alpha_{(n-1) 2} & \cdots & \alpha_{(n-1)(n-1)} & \alpha_{(n-1) n} \\
\alpha_{n 1} & \alpha_{n 2} & \cdots & \alpha_{n(n-1)} & \alpha_{n n}
\end{array}\right]\left[\begin{array}{c}
F_{1} \\
F_{2} \\
\vdots \\
F_{n-1} \\
F_{n}
\end{array}\right] .
$$

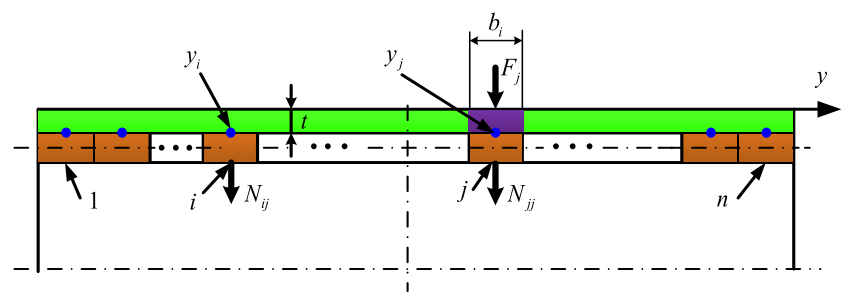

Fig. 4. Schematic diagram of channels coupling mechanism (Online version in color.) 
This matrix form can be abbreviated as

$$
\mathbf{N}=\mathbf{A F} \text {. }
$$

If the radial pressure received by the measuring roll is $F_{j}(j=1 \sim n)$, the force detected by the sensor will become $N_{j}(j=1 \sim n)$ due to the coupling of the channels. If we do not decouple the channels and assume $\mathbf{N}=\mathbf{F}$, it will cause significant measurement error. Equations (6) and (7) are coupling matrix equations, and the coupling matrix coefficients can be obtained by theoretical calculation or flatness experimental calibration.

Equation (3) shows that the influence coefficient of any channel to itself is a constant.

$$
\beta_{i i}=\operatorname{const}(i=1 \sim n) .
$$

In the theoretical calculation, stiffness of each sensor can be considered to be equal.

$$
k_{1}=k_{2}=\cdots=k_{n-1}=k_{n}=1 / \beta_{i i} .
$$

Now, the coupling matrix A becomes a symmetric matrix in which the diagonal entries are equal to one.

\subsection{Analysis of Coupling Channels Instance and Decoupling Model}

A measuring roll of a $1450 \mathrm{~mm}$ six-high cold rolling mill has a total of 57 channels, and each channel has a width of $26 \mathrm{~mm}$. The main technical parameters of the flatness meter are shown in Table 1. Pressure distribution and flatness distribution of the measuring roll are shown in Fig. 5 according to the theoretical calculation of the channel coupling coefficients, wherein a uniform pressure of 100 $\mathrm{N} / \mathrm{mm}$ is applied to all channels. The measured pressure is

Table 1. Main parameters of 1450 flatness meter.

\begin{tabular}{cc}
\hline Parameters & Value \\
\hline Measuring roll diameter $(\mathrm{mm})$ & 300 \\
Roll length (mm) & 1500 \\
Measuring unit width (mm) & 26 \\
Channel of measuring units & 57 \\
Effective detection length (mm) & 1482 \\
Roll surface hardness & HRC 61-63 \\
Roll surface roughness & Ra 0.4 \\
\hline
\end{tabular}

approximately 2.5 times higher than the actual pressure, and the flatness distribution is also significantly different. The measured coupling pressure is significantly lower at both ends than in the middle part. In fact, the uniform pressure corresponds to perfect flatness, i.e., the flatness value of each channel is 0 . However, due to the channel coupling effect, the detected flatness has obvious bilateral waves, and the error is approximately 4 I-Units. If flatness closed-loop control system is adjusted according to bilateral waves and bending force is increased, a middle wave in physical strip will be inevitable.

In production practice, flatness distribution is generally decomposed into four components. Roll tilting is adjusted to correct linear term of the defects. Then, bending roll is adjusted to correct quadratic term of the defects. Then, segmental cooling and asymmetric bending roll or shifting roll are adjusted to correct cubic and quartic term of defects. To analyze the coupling measurement error of each order of flatness, an uniform pressure is applied to the measuring roll one to four times as those four components mentioned above. The expression is

$$
\left\{\begin{array}{l}
p_{1}=100+100 \bar{y} \\
p_{2}=100+100\left(3 \bar{y}^{2}-1\right) / 2 \\
p_{3}=100+100\left(5 \bar{y}^{3}-3 \bar{y}\right) / 2 \\
p_{4}=100+100\left(35 \bar{y}^{4}-30 \bar{y}^{2}+3\right) / 8
\end{array}\right.
$$

In the formula, $\bar{y}=2 y / L_{\mathrm{ef}}$ and the coordinates are normalized. The value equals 0 at the center point of the roll body in the axial direction. The variation range is $[-1,1]$.

Figure 6 shows that when the pressure distribution is applied as linear term, the pressure distribution from measuring becomes larger as a whole due to the coupling of the channels. The downward tendency tends to occur at the edges where the force is larger. At the less stressed edge, the flatness is 5 I-Units smaller than the actual flatness. The flatness is approximately $10 \mathrm{I}$-Units larger than the actual flatness at the edge with a larger force.

Figure 7 shows that the pressure distribution at both edges is lower than the actual distribution when the distribution pressure is applied as a quadratic term. The flatness at the middle part of the roll body is 3 I-Units smaller than the actual flatness. The flatness at both edges is approximately 10 I-Units larger than the actual flatness. The flatness at

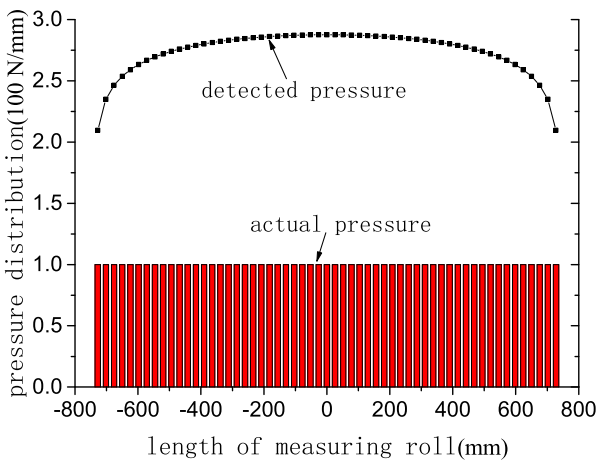

(a) pressure distribution

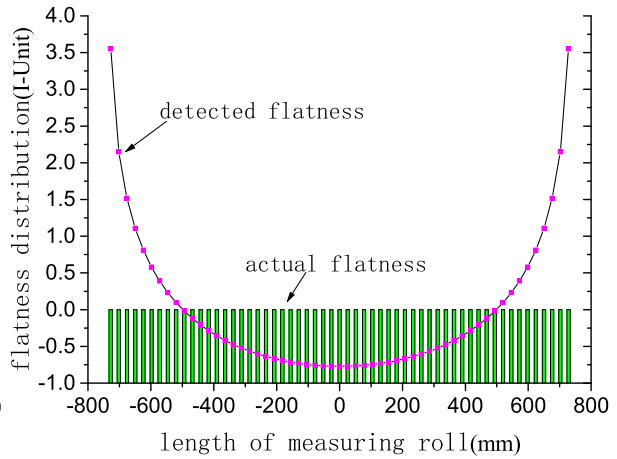

(b) flatness distribution

Fig. 5. Detected coupling pressure and flatness distribution when uniform force is applied. (Online version in color.) 


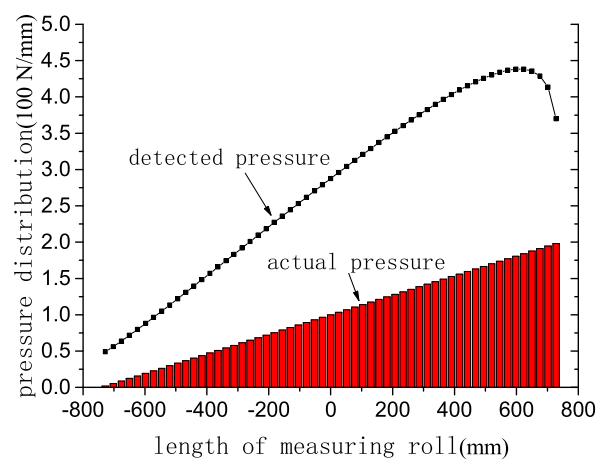

(a) pressure distribution

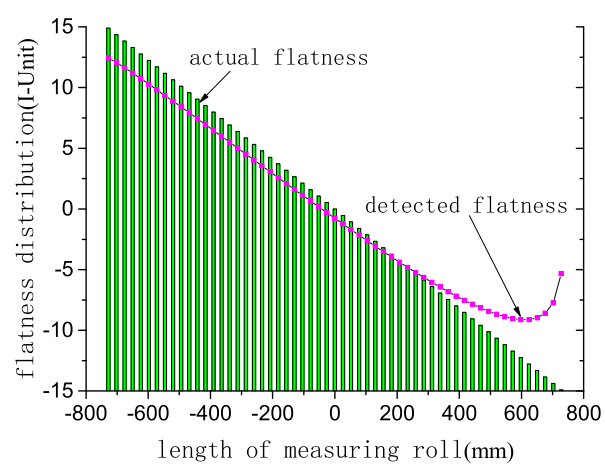

(b) flatness distribution

Fig. 6. Detected coupling pressure and flatness distribution when applying load as linear term. (Online version in color.)

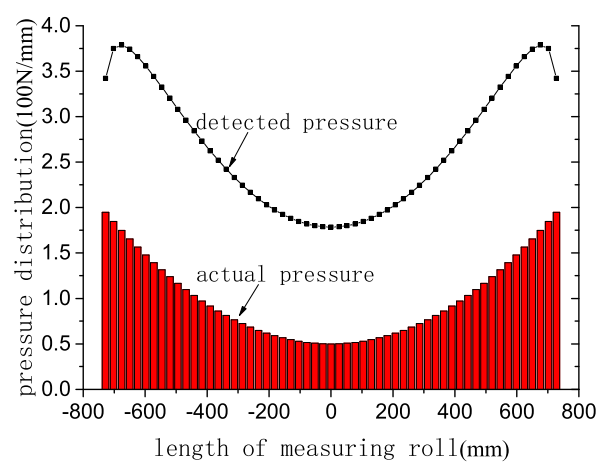

(a) pressure distribution

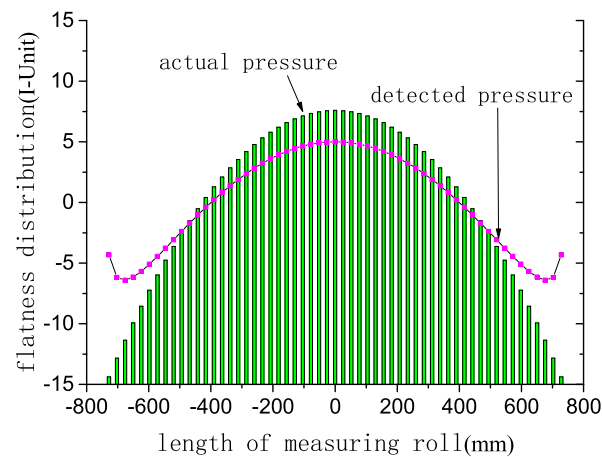

(b) flatness distribution

Fig. 7. Detected coupling pressure and flatness distribution when applying load as a quadratic term. (Online version in color.)

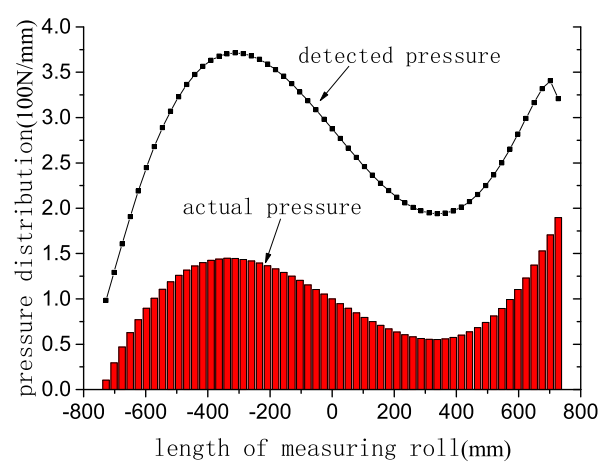

(a) pressure distribution

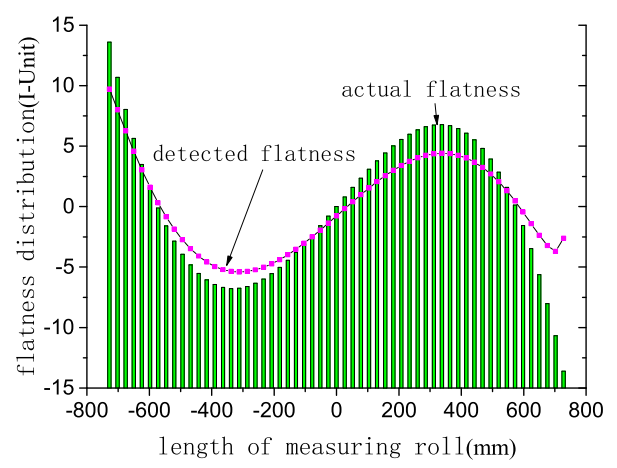

(b) flatness distribution

Fig. 8. Detected coupling pressure and flatness distribution when applying load as a cubic term. (Online version in color.)

one-quarter of the length on both sides is not substantially different from the actual flatness.

Figure 8 shows that when the pressure distribution is applied as a cubic term, the pressure distribution at the right edge of the roll has a significant downward tendency compared with the actual distribution. The measured flatness at the left edge of the roll is approximately 3.5 I-Units smaller than the actual flatness. The flatness at the right edge is approximately $10 \mathrm{I}-$ Units larger than the actual flatness. The flatness at one-quarter of the length on the left is 2.5 I-Units larger than the actual flatness. The flatness at onequarter of the length on the right is 3 I-Units smaller than the actual flatness.
Figure 9 shows that when the pressure distribution is applied as a quartic term, the measured pressure distribution at both edges has a small degree of downward turn compared with the actual distribution. The flatness at both edges is approximately $13 \mathrm{I}$-Units larger than the actual flatness, and the flatness at the middle part of the measuring roll is not substantially different from the actual flatness. The flatness at one-quarter of the length on both sides is approximately 2.5 I-Units smaller than the actual flatness.

In summary, when different distribution pressures are applied to the measuring roll, significant flatness measuring errors are caused by the coupling effect of the channels. The magnitude and direction of errors caused by different 


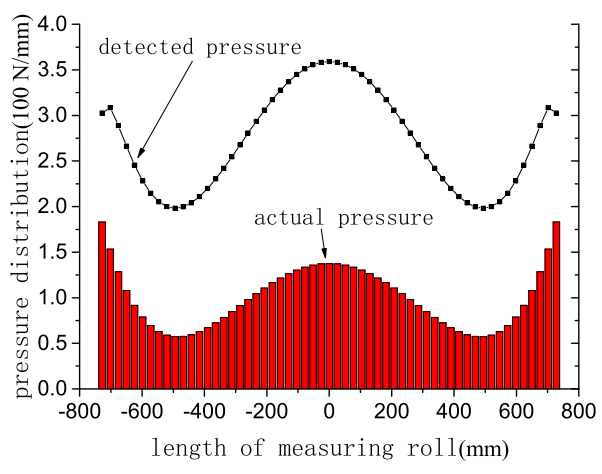

(a) pressure distribution

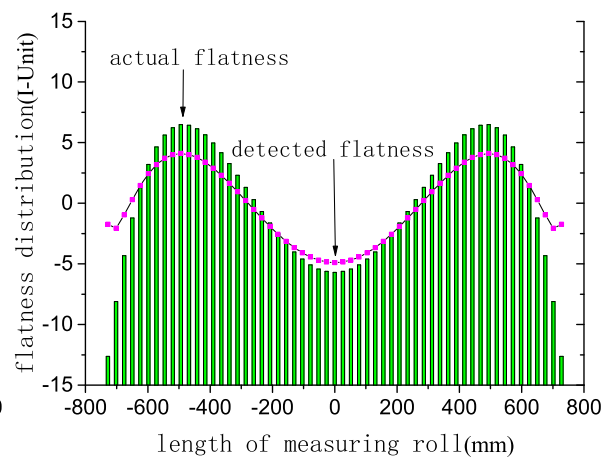

(b) flatness distribution

Fig. 9. Detected coupling pressure and flatness distribution when applying load as a quartic term. (Online version in color.)
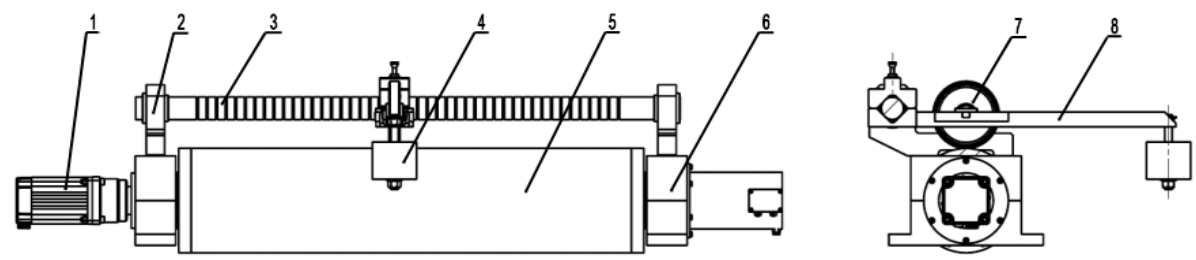

(1) Motor, (2) brackets, (3) beam, (4) weights, (5) measuring roll, (6) bearing seats,

(7) pressure wheel, (8) loading rods

Fig. 10. Calibration device of flatness measuring roll.

distributed pressures are also different. It is difficult to summarize a simple correction rule. To decouple the measuring channels, Eqs. (6) and (7) can be transformed to

$\left[\begin{array}{c}F_{1} \\ F_{2} \\ \vdots \\ F_{n-1} \\ F_{n}\end{array}\right]=\left[\begin{array}{ccccc}\alpha_{11} & \alpha_{12} & \cdots & \alpha_{1(n-1)} & \alpha_{1 n} \\ \alpha_{21} & \alpha_{21} & \cdots & \alpha_{2(n-1)} & \alpha_{2 n} \\ \vdots & \vdots & \vdots & \vdots & \vdots \\ \alpha_{(n-1) 1} & \alpha_{(n-1) 2} & \cdots & \alpha_{(n-1)(n-1)} & \alpha_{(n-1) n} \\ \alpha_{n 1} & \alpha_{n 2} & \cdots & \alpha_{n(n-1)} & \alpha_{n n}\end{array}\right]^{-1}\left[\begin{array}{c}N_{1} \\ N_{2} \\ \vdots \\ N_{n-1} \\ N_{n}\end{array}\right]$.

Equation (11) can be rewritten as

$$
\mathbf{F}=\mathbf{A}^{-1} \mathbf{N}
$$

Equations (11) and (12) are named decoupling matrix equations, and $\mathbf{A}^{-1}$ is decoupling matrix.

Equation (11) shows that if all the entries of the coupling matrix $\mathbf{A}$ are known, the inverse matrix, which is the decoupling matrix $\mathbf{A}^{-1}$, can be obtained. Then, $\mathbf{A}^{-1}$ can be multiplied by detection force vector $\mathbf{N}$ to obtain real force vector $\mathbf{F}$. The force $\mathbf{F}$ applied to the roll surface calculated by decoupling can be obtained according to Eq. (11). However, there are still differences between the calculated and actual values, and the stiffness of each sensor is considered constant during the above theoretical analysis. In addition, the fact that the measuring roll is considered as an elastic half-space does not completely match actual situation. Therefore, to obtain an accurate coupling matrix, a measuring roll calibration experiment is required.

\section{Experimental Calibration of the Coupling Channels}

The WRS flatness meter calibration device is shown in

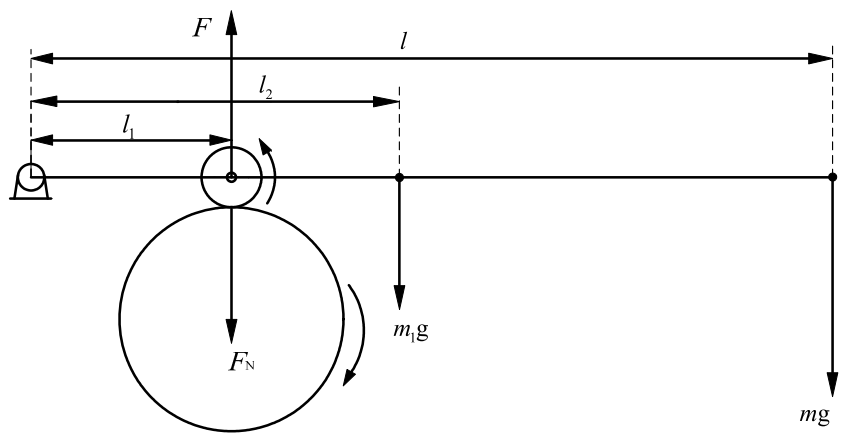

Fig. 11. Mechanics model of calibration device.

Fig. 10. The transmission motor drives the rotational motion of the measuring roll. Together, the loading rod, the beam, the bracket, the pressure roller and the weight form a loading mechanism. The pressure roller is pressed at the surface of the measuring roll on the top. The loading mechanics model is shown in Fig. 11, and the radial pressure (calibration force) $F$ obtained by the measuring roll is

$$
F=\frac{m_{1} g l_{2}+m g l}{l_{1}} \text {. }
$$

where $m$ is the mass of the weight, which is an integral multiple of $9.20 \mathrm{~kg} ; m_{1}$ is the mass of the loading rod and the pressure wheel, which is $7.33 \mathrm{~kg} ; g$ is the gravitational acceleration; $l=800 \mathrm{~mm} ; l_{1}=200 \mathrm{~mm}$; and $l_{2}=310 \mathrm{~mm}$.

The measuring roll of the $1450 \mathrm{~mm}$ six-high cold rolling mill was experimentally calibrated by the above mentioned calibration device. The first channel to the 57th channel were sequentially loaded, and the values of all the measuring forces of the channels were recorded when each channel was loaded. Taking the intermediate channel (29th channel) 
loading process as an example, a comparison between the theoretical coupling coefficients and the experimental coupling coefficients is shown in Fig. 12. The channels coupling phenomenon does exist during the actual loading process, but the theoretically calculated coupling coefficients is larger than the experimental calibration value. The main reason of error is the difference between the elastic half-space hypothesis and the actual situation. Later, finite element method can be used for more accurate modeling calculations.

When a channel is stressed, the coupling coefficients of

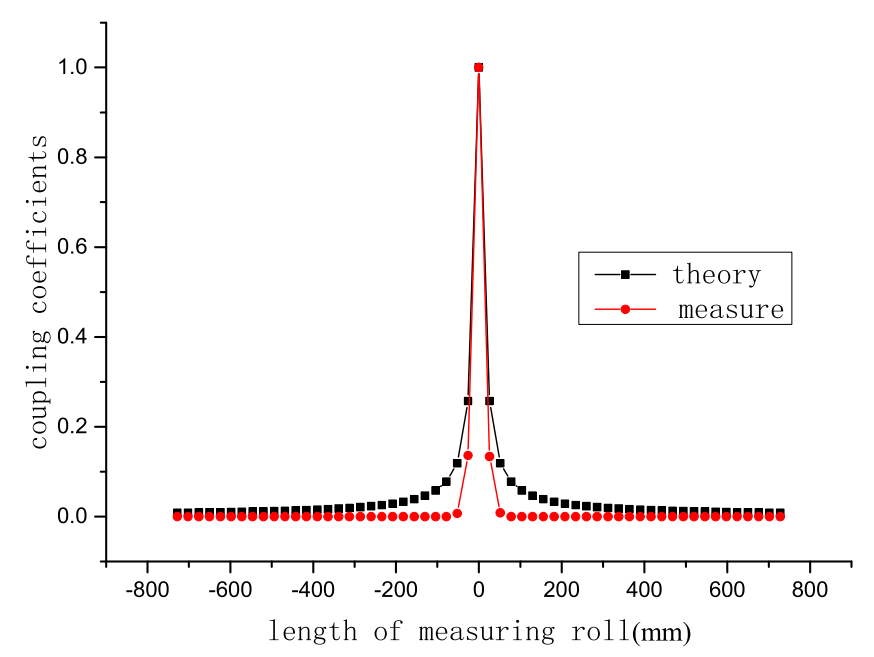

Fig. 12. Comparison of theoretical and experimental coupling coefficients. (Online version in color.)

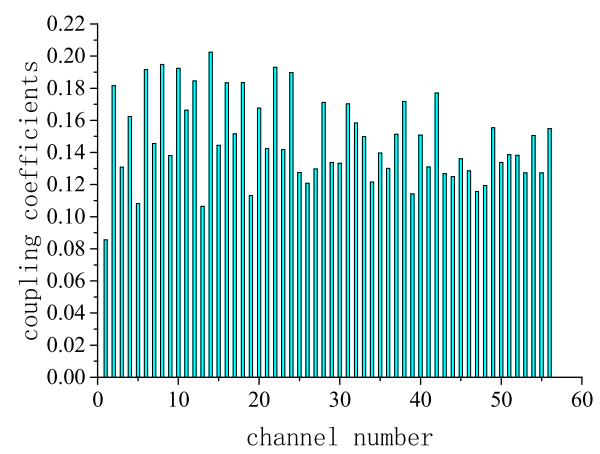

(a)Coupling coefficients $\alpha_{(i-1) i}$

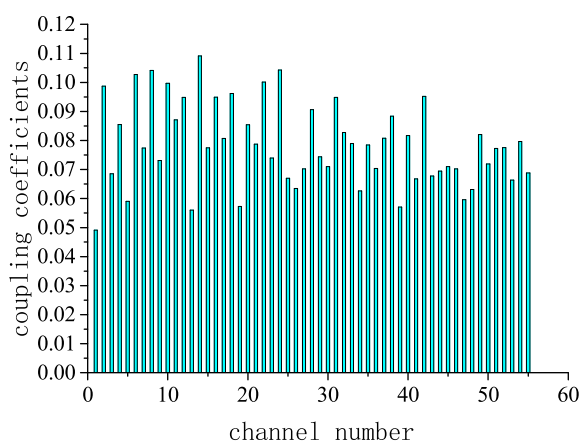

(c) Coupling coefficients $\alpha_{(i-2) i}$ the first left and right adjacent channels are approximately 0.15 , the coupling coefficients of the second left and right channels are approximately 0.008 , and the coupling coefficients of other channels are approximately zero. In practical applications, only the effects on the first left and right adjacent channels and the second left and right channels are considered. Therefore, only the experimental calibration results of the coupling coefficients, $\alpha_{(i-1) i}(i=2-57), \alpha_{(i+1) i}(i=1-56)$, $\alpha_{(i-2) i}(i=3-57)$ and $\alpha_{(i+2) i}(i=1-55)$, are given, as shown in Fig. 13. The figure shows that the coupling coefficients

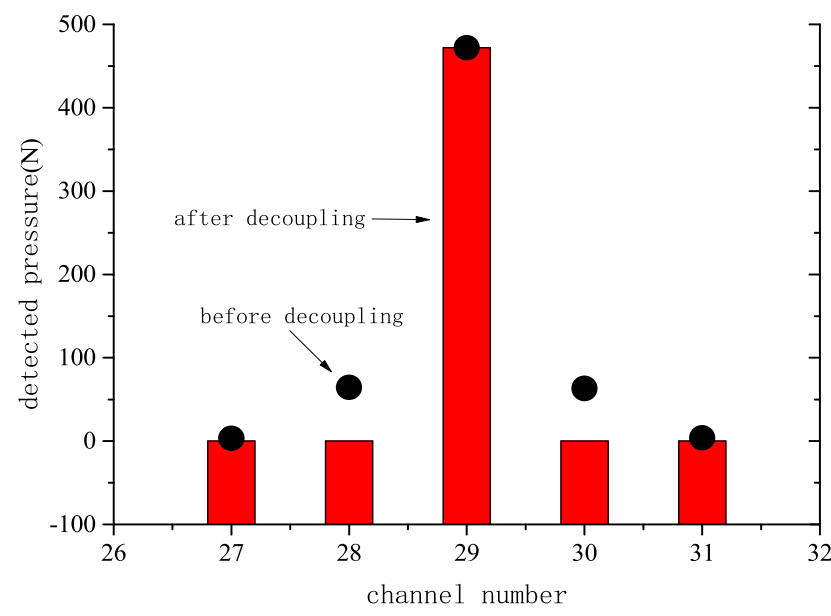

Fig. 14. Comparison of the measuring force before and after decoupling of each channel when applying load in the middle channel. (Online version in color.)

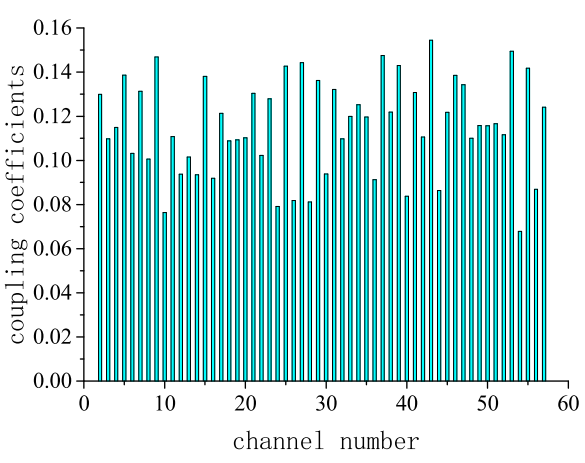

(b) Coupling coefficients $\alpha_{(i+1) i}$

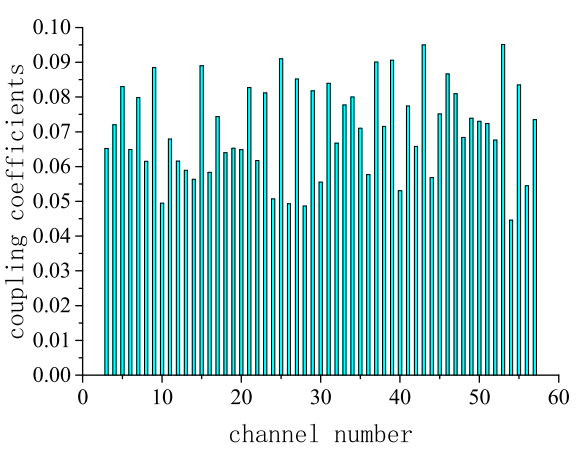

(d) Coupling coefficients $\alpha_{(i+2) i}$

Fig. 13. Coupling coefficients of calibration. (Online version in color.) 
of different channels have a certain degree of fluctuation. The main reasons for these fluctuations are machining errors in the sensor mounting hole, installation errors in the axial position of the sensors, and deviations in the interference of different sensors installation. Therefore, it is difficult to calculate the coupling coefficients from a completely theoretical model while experimental calibration can be easy to obtain authentic results.

The intermediate channel (29th channel) loading process is again taken as an example; according to the coupling coefficients of Eq. (11) and experimental calibration, the measuring results before and after decoupling are shown in Fig. 14. The channel coupling phenomenon is nearly eliminated by decoupling process, which shows the necessity of the theoretical model and experimental calibration.

\section{Industrial Application}

The $1450 \mathrm{~mm}$ six-high cold rolling mill flatness meter, as mentioned above, was applied to an industrial site in May 2018. The $1450 \mathrm{~mm}$ mill parameters and rolling parameters are shown in Table 2. For an SPCC rolled strip material with a thickness of $0.34 \mathrm{~mm}$ and a width of $1000 \mathrm{~mm}$, the pressure and flatness of two detection examples before and after decoupling are shown in Fig. 15. The pressure distributions before and after decoupling are very different. The average pressure before decoupling is approximately 1.3 times that after decoupling. The coupling pressure distribution shows a downward turning trend on both sides of the strip. Because the coupling coefficients fluctuate within

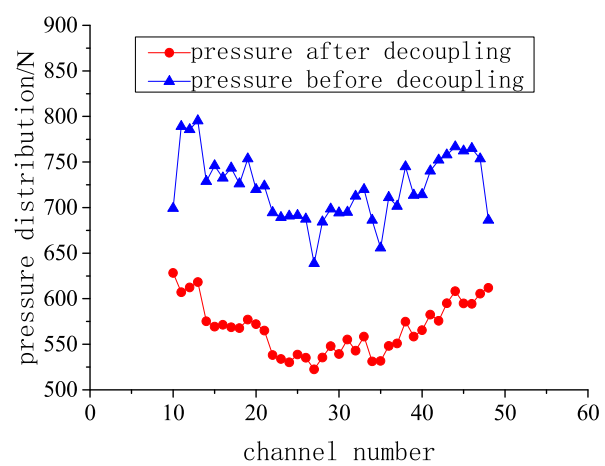

(a) Example 1 pressure comparison

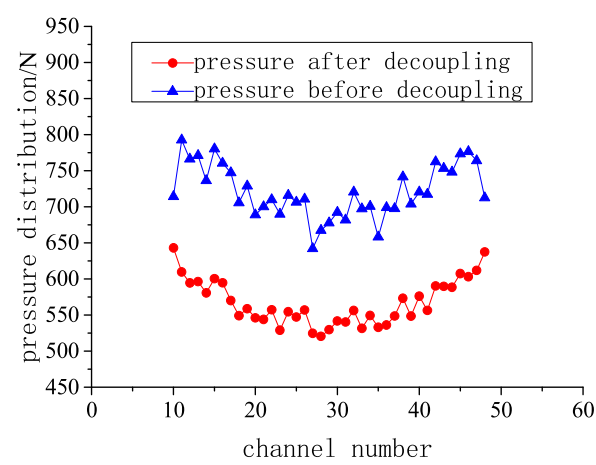

(c) Example 2 pressure comparison a certain range, the coupling pressure distribution also has obvious fluctuations. After decoupling, the pressure distribution becomes smoother and closer to the actual state. The differences in the flatness before and after decoupling are mainly shown on the two sides of the strip. Before decoupling, there are both middle waves and side waves. After decoupling, there is only a simple middle wave. The flatness distribution before decoupling has larger fluctuations, while the decoupled flatness distribution is smoother.

In actual production, the target flatness of strip product from this mill is microscopic middle waves. The flatness defect cannot be completely eliminated in a subsequent continuous annealing processing line (CAPL) if the middle waves are large. If the decoupling process is not performed, the flatness control system will consider that there are waves

Table 2. Mill parameters and rolling parameters

\begin{tabular}{cc}
\hline parameters & value \\
\hline Tension range $(\mathrm{KN})$ & $10-120$ \\
wrap angle $\left({ }^{\circ}\right)$ & 8 \\
Max rolling force $(\mathrm{KN})$ & 20000 \\
Strip width range $(\mathrm{mm})$ & $800-1250$ \\
Final strip thickness range $(\mathrm{mm})$ & $0.25-2.5$ \\
Work roll diameter $(\mathrm{mm})$ & $280-330$ \\
Work roll length $(\mathrm{mm})$ & 1450 \\
Max rolling speed $(\mathrm{m} / \mathrm{min})$ & 800 \\
\hline
\end{tabular}

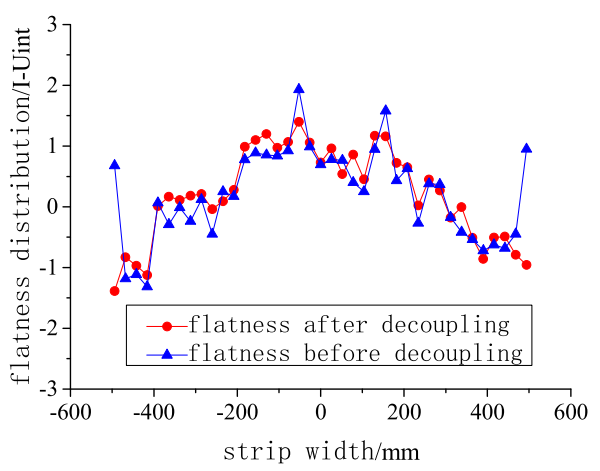

(b) Example 1 flatness comparison

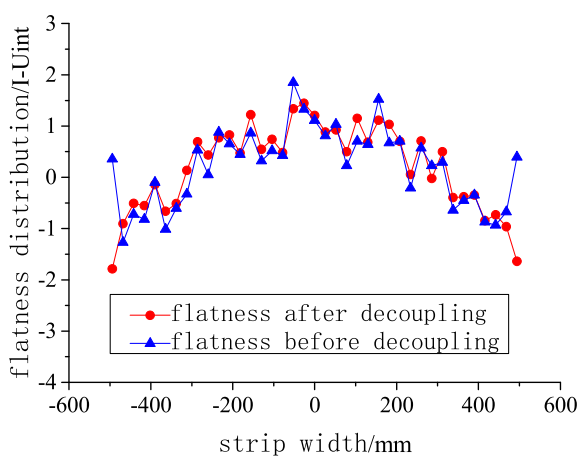

(d) Example 2 flatness comparison

Fig. 15. Pressure and flatness comparison before and after decoupling. (Online version in color.) 


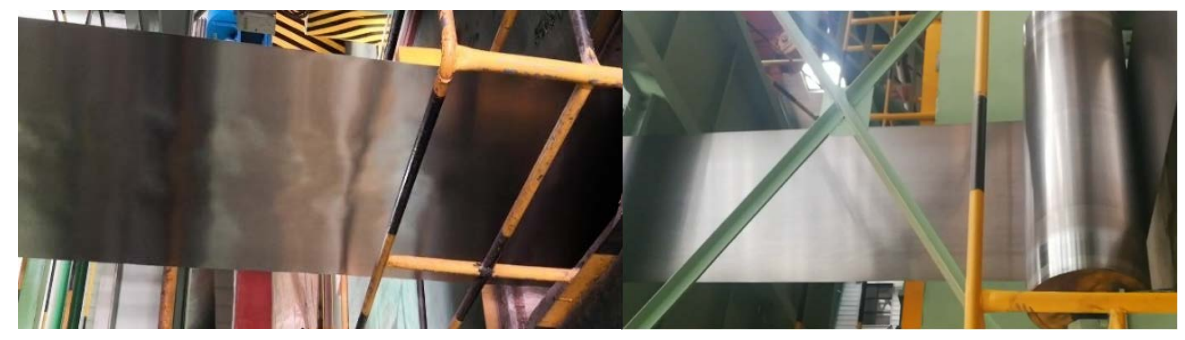

(a) Flatness before decoupling process

(b) Flatness after decoupling process

Fig. 16. Physical flatness of the strip before and after decoupling process. (Online version in color.)

on both sides of the strip, then the bending force will continuously increase, eventually causing physical flatness to be excessively large. After decoupling is applied, the flatness control system will accurately decrease the difference between target flatness and measuring flatness. The actual flatness is an approximately flat microscopic middle wave, which achieves the flatness control target. The flatness control results before and after decoupling are shown in Fig. 16. The comparison of Figs. 15 and 16 shows that the flatness after decoupling is consistent with the physical flatness.

\section{Conclusions}

(1) When a load is applied to a certain measuring channel of the whole-roller seamless flatness meter, the signal of this channel will change and the signal of the adjacent channels will be significantly interfered, causing flatness measuring errors. If the flatness channel coupling effect is not considered, when the physical flatness is completely flat, the measured flatness will be a bilateral wave, which is very different from the actual situation.

(2) When different distribution forces act on the measuring roll, significant flatness measuring errors will occur due to the coupling of the channels. The magnitude and direction of the errors are also different. Because of the difference between the elastic half-space hypothesis and actual situation, the theoretically calculated coupling coefficient is larger than the coupling coefficient from experimental calibration.

(3) After decoupling by the decoupling matrix equation, the flatness measuring error can be eliminated. After decoupling, the detected flatness is quite close to the physical flatness, which is essential for controlling flatness of strips.

\section{REFERENCES}

1) L. P. Xu, L. You, D. H. Wang and X. T. Su: China Metall., 24 (2014), 23 (in Chinese).

2) X. G. Liang, Z. J. Jiao, G. D. Wang, X. H. Liu, J. F. Guo, H. T. Li and G. Wang: Metall. Equip., 6 (2006), 36 (in Chinese).

3) L. J. Chen, B. Han, W. Tan, A. P. Ruan and S. Z. Wang: Steel Roll., 29 (2012), 38 (in Chinese).

4) B. Guan, Y. Zang, X. H. Han and K. Zheng: Sensors, 18 (2018), 1638.

5) D. C. Tran, N. Tardif and A. Limam: Int. J. Solids Struct., 69-70 (2015), 343.
6) Y. Isei, T. Kato, M. Osugi and T. Ohta: Tetsu-to-Hagané, 105 (2019), 20 (in Japanese).

7) S. Abdelkhalek, P. Montmitonnet, M. Potier-Ferry, H. Zahrouni, N. Legrand and P. Buessler: Ironmaking Steelmaking, 37 (2010), 290.

8) L. P. Yang, H. X. Yu, R. M. Li and W. Yuan: Ironmaking Steelmaking, 45 (2018), 457.

9) L. P. Yang, H. X. Yu, D. C. Wang, Z. Zhang and Z. Y. Jiang: Steel Res. Int., 88 (2017), 1600383.

10) H. M. Liu, B. Q. Yu, L. P. Yang, D. Ding, H. X. Yu and S. W. Chen: A Type of Seamless and Embedded Signal Processor Used for Shapemeter, China Patent CN105005287A, (2015).

11) H. M. Liu, B. Q. Yu, L. P. Yang and Y. Peng: Iron Steel, 46 (2011), 86 (in Chinese).

12) H. M. Liu, J. Liu, B. Q. Yu, L. Q. Yang and Y. Zhang: J. Mech. Eng., 53 (2017), 87 (in Chinese).

13) D. C. Wang, H. M. Liu and J. Liu: Chin. J. Mech. Eng., 30 (2017), 1248.

14) L. He, J. Wang and F. Zhang: J. Iron Steel Res., 25 (2013), 58 (in Chinese).

15) X. Y. Shan: Ph.D. thesis, Yanshan University, (2011), 6, http:// kns.cnki.net $/ \mathrm{KCMS} /$ detail/detail.aspx dbcode $=\mathrm{CDFD} \& \mathrm{dbname}=\mathrm{C}$ DFD0911\&filename $=1011281536$. nh\&uid = WEEvREcwSIJHSIdR a1FhdkJkVG1BdXBwUCtzWGhOTzFWY2F6UzVLYVllcz0=\$9A 4hF YAuvQ5obgVAqNKPCYcEjKensW4IQMovwHtwkF4VYPoHbKxJw!!\&v=Mjg0NjR1eFITN0RoMVQzcVRyV00xRnJDVVJMT2ZZ T1JuRnkzbFZiN09WRjI2SDdHd0g5VFBxWkViUEISOGVYMUw=, (accessed 2019-07-26) (in Chinese).

16) D. Weisz-Patrault: Int. J. Solids Struct., 56-57 (2015), 175.

17) J. W. Liu, D. H. Zhang, J. S. Wang and P. F. Wang: J. Iron Steel Res. Int., 17 (2010), 35.

$18)$ B. Q. Yu, Y. B. Sun, H. M. Liu, L. You and Y. Peng: J. Iron Steel Res. Int., 17 (2010), 21.

19) R. M. Li, L. P. Yang, B. Q. Yu, H. M. Liu and H. X. Yu: Iron Steel, 48 (2013), 41 (in Chinese).

20) R. M. Li, L. P. Yang, B. Q. Yu, H. M. Liu and C. Z. Du: Iron Steel, 48 (2013), 40 (in Chinese)

21) Z. X. Zhao, D. C. Wang, P. F. Wang and H. M. Liu: Iron Steel, 50 (2015), 49 (in Chinese).

22) L. P. Yang, B. Q. Yu, D. Ding and H. M. Liu: J. Cent. South Univ., 19 (2012), 994.

23) L. P. Yang, B. Q. Yu, R. M. Li, H. M. Liu and C. Z. Du: Chin. J. Mech. Eng., 50 (2014), 30.

24) H. M. Wu, H. M. Liu and K. Wang: Int. J. Adv. Manuf. Technol., 94 (2018), 3189.

25) P. F. Wang, D. H. Zhang, J. W. Liu, J. S. Wang and X. F. Yu: Chin. J. Mech. Eng., 47 (2011), 58.

26) J. C. Lian and H. M. Liu: Thicness and Shape Control for Rolling Strip, Weapons Industry Press, Beijing, (1996), 106 (in Chinese).

27) V. B. Ginzburg: High-Quality Steel Rolling: Theory and Practice, CRC Press, Boca Raton, FL, (1993), 489.

28) H. M. Liu, B. Q. Yu, L. P. Yang, Y. Peng, D. Ding, Z. M. Li and Q. Fu: A Whole Roll Seamless Wireless Flatness Meter, China Patent CN101694368B, (2011).

29) H. M. Liu: Three Dimensional Rolling Theory and Its Application, Science Press, Beijing, (1999), 293 (in Chinese).

30) K. L. Johnson: Contact Mechanics, Cambridge University Press, Cambridge, UK, (1985), 10.

31) D. C. Wang, Y. L. Wu and H. M. Liu: Iron Steel, 50 (2015), 37 (in Chinese). 